\title{
Relaxation bottleneck and its suppression in semiconductor microcavities
}

\author{
A. I. Tartakovskii, ${ }^{1,2}$ M. Emam-Ismail,${ }^{2}$ R. M. Stevenson, ${ }^{2}$ M. S. Skolnick, ${ }^{2}$ V. N. Astratov, ${ }^{2}$ D. M. Whittaker, ${ }^{3}$ \\ J. J. Baumberg, ${ }^{4}$ and J. S. Roberts ${ }^{5}$ \\ ${ }^{1}$ Institute of Solid State Physics, RAS, 142432 Chernogolovka, Russia \\ ${ }^{2}$ Department of Physics and Astronomy, University of Sheffield, Sheffield S3 7RH, United Kingdom \\ ${ }^{3}$ Toshiba Research Europe Ltd., Cambridge CB4 4WE, United Kingdom \\ ${ }^{4}$ Department of Physics and Astronomy, University of Southampton, Southampton SO17 1BJ, United Kingdom \\ ${ }^{5}$ Department of Electronic and Electrical Engineering, University of Sheffield, Sheffield S1 3JD, United Kingdom
}

(Received 6 March 2000; revised manuscript received 18 April 2000)

\begin{abstract}
A polariton relaxation bottleneck is observed in angle-resolved measurements of photoluminescence emission from a semiconductor microcavity. For low power laser excitation, low $k$ polariton states are found to have a very small population relative to those at high $k$. The bottleneck is found to be strongly suppressed at higher powers in the regime of superlinear emission of the lower polariton states. Evidence for the important role of carrier-carrier scattering in suppression of the bottleneck is presented.
\end{abstract}

Semiconductor microcavities (MC's) with embedded quantum wells (QW's) have attracted great interest recently. In these structures, two-dimensional (2D) confinement of photon and exciton modes is realized. In high finesse MC's, as a result of exciton-photon coupling, mixed $2 \mathrm{D}$ polariton states occur ${ }^{1}$ which exhibit a strong dependence of their properties on their relative exciton and photon contents. ${ }^{2}$ In particular, due to the very small in-plane photon mass in MC's $\left(M_{p} \approx 10^{-4} m_{e x}\right)$, polaritons with a significant photon fraction have a very steep $E-k$ dispersion curve, ${ }^{3}$ and a density of states considerably smaller than that of excitons in a bare QW.

Of particular relevance to the present work, energy relaxation in the polariton system is expected to be strongly modified relative to that of uncoupled excitons. Acoustic phonon scattering rates from high $k$ exciton states into polariton states with wave vectors corresponding to the strong coupling regime $\left(k \leqslant 5-6 \times 10^{4} \mathrm{~cm}^{-1}\right)$ are expected to be small due to the large energy transfer required compared to typical acoustic phonon energies of $1 \mathrm{meV}$. Furthermore, the strongly coupled states have very short radiative lifetime $(\sim 1 \mathrm{psec})$ due to their high photon fraction, thus leading to a nonthermal polariton population and the occurrence of a relaxation bottleneck. Such bottleneck effects have been much discussed theoretically for the cavity polariton system. ${ }^{4,5}$ References 4 and 5 calculate the expected population distributions for both upper and lower polariton branches, including exciton-phonon and exciton-exciton scattering. Very large depletions of the low $k$ states, relative to those at high $k$, by factors up to $10^{4}$, are predicted. Such relaxation bottlenecks also occur in bulk semiconductors, ${ }^{6}$ but are very difficult to study since the polaritons do not decay directly into external photons. In MC's, by contrast, direct polariton decay does occur due to photon leakage out of the cavity, permitting direct study of the population distribution. ${ }^{7}$

A manifestation of the bottleneck has been observed in time-resolved measurements of the polariton emission in II-VI based microcavities. ${ }^{8}$ The authors found an abrupt decrease of the polariton PL decay time when the character of the polariton states changed from photonlike to excitonlike.
This observation indicates a slowdown of the relaxation process into polariton states with high photon fraction.

In this paper, we present a definitive observation of the relaxation bottleneck for lower branch polaritons (LP's) from angle resolved photoluminescence (PL). Nonresonant cw laser excitation creates excitons at high energy which relax to form a reservoir of high $k\left(>10^{5} \mathrm{~cm}^{-1}\right)$ excitons. These excitons scatter into the observable polariton states and give rise to the PL. For low density excitation, low $k(<1.5$ $\times 10^{4} \mathrm{~cm}^{-1}$ ) occupancies nearly an order of magnitude smaller than those at higher $k$ are found. Strong redistribution of the polariton population, and suppression of the bottleneck, is found in the regime where the LP emission exhibits superlinear behavior with increasing excitation intensity ${ }^{9}$. Detailed measurements performed in a wide temperature range demonstrate that the efficiency of the scattering into the LP states is very sensitive to temperature and energy separation from the exciton reservoir. This observation, in combination with the deduction of a new scattering process at higher excitation densities, indicates the important role of exciton-exciton scattering in suppressing the bottleneck.

The sample studied is a high quality single MC structure grown by metal organic vapor phase epitaxy. The Bragg reflectors are composed of 17 (20) repeats of $\lambda / 4$ $\mathrm{Al}_{0.13} \mathrm{Ga}_{0.87} \mathrm{As} / \mathrm{Al}$ As layers in the top (bottom) mirrors. The $3 / 2 \lambda$ GaAs cavity contains six $10-\mathrm{nm} \mathrm{In}_{0.06} \mathrm{Ga}_{0.94} \mathrm{As} / \mathrm{GaAs}$ QW's. The vacuum Rabi splitting was $\Omega \approx 6 \mathrm{meV}$, with linewidths of $<1 \mathrm{meV}$. Nonuniformities of the cavity width permit tuning of the photon mode energy over a wide range of negative and positive detunings $\left(\Delta=E_{c a v}-E_{e x}\right)$. Measurements were carried out with the sample immersed either in superfluid $\mathrm{He}$ or in He gas. A Ti-sapphire laser was used for nonresonant $(1.56 \mathrm{eV})$ excitation. The PL signal was collected by a lens which imaged the excited sample region (collection angle $<2^{\circ}$ ) on to a fiber. Both the lens and the fiber were mounted on a rotating rail permitting precise angle tuning. The signal was detected by a single monochromator/ CCD system.

PL spectra for a detuning of $\Delta=-4.3 \mathrm{meV}$ and temperature $T=34 \mathrm{~K}$ over a wide range of collection angles $(\Phi$, 


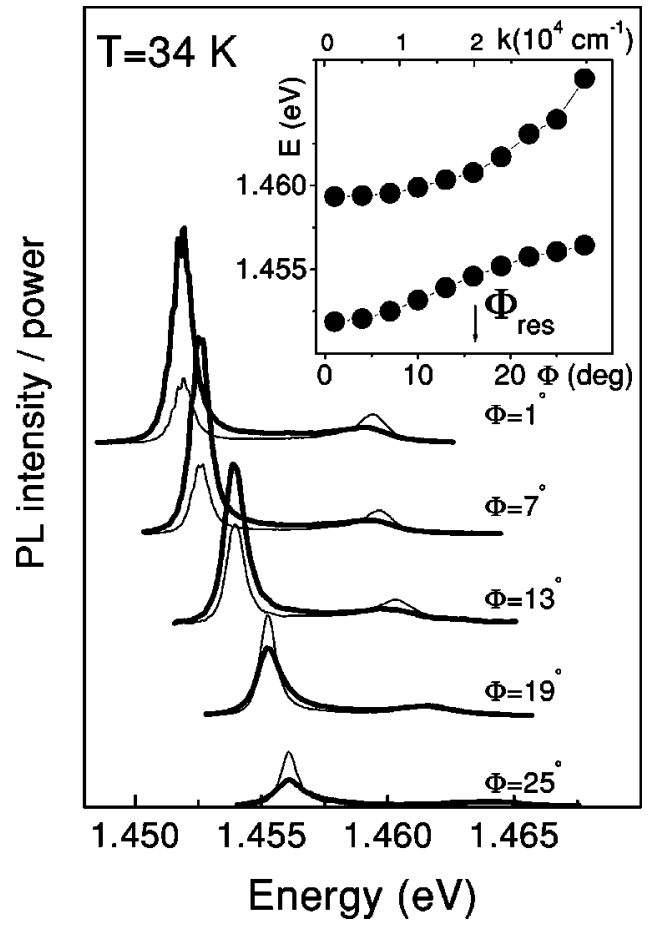

FIG. 1. Angle-resolved PL spectra recorded for $\Delta=-4.3 \mathrm{meV}$ at $T=34 \mathrm{~K}$. The spectra shown by thick (thin) lines are collected at excitation density $P=80(P=5) \mathrm{W} / \mathrm{cm}^{2}$ using nonresonant cw laser excitation. The inset shows the measured polariton dispersion.

measured from the normal to the sample), for excitation powers of $P=80$ (thick lines) and $P=5$ (thin lines) W/ $\mathrm{cm}^{2}$, are presented in Fig. 1. The spectra are normalized to the excitation intensity. At low $P$, the LP emission is found to peak around $\sim 16^{\circ}$, corresponding to resonance between the photon and exciton modes $\left(\Phi_{\text {res }}\right)$, as seen by reference to the measured polariton dispersion in the inset. By contrast, at high $P$, the PL intensity peaks around $\Phi=0$ and decreases continuously with increasing angle. Furthermore, at small $\Phi$ a strong superlinear growth of the LP emission is observed with increasing $P$, as shown in Fig. 2(a). With increasing $\Phi$, the superlinear behavior becomes much weaker, and eventually for $\Phi>\Phi_{\text {res }}$, the PL intensity varies sublinearly with $P$. From 5 to $80 \mathrm{~W} / \mathrm{cm}^{2}$, the LP peak shifts by less than 0.1 $\mathrm{meV}$, showing that the system is always in the strong coupling regime.

The normalized integrated intensities from Fig. 1 are plotted against $\Phi$ (and in-plane wave vectors, since $k$ $=\omega / c \sin \Phi)$ in Fig. 2(b), together with results for several intermediate powers. The key features of Fig. 1, the initial increase in PL intensity with $\Phi$ for $P$ up to $20 \mathrm{~W} / \mathrm{cm}^{2}$, the decrease from $\Phi=0$ to $\Phi \sim 30^{\circ}$ for $P \geqslant 40 \mathrm{~W} / \mathrm{cm}^{2}$, and the strong superlinear increase with $P$ for $\Phi<\Phi_{\text {res }}$, are clearly seen. For larger angles, $\Phi>\Phi_{\text {res }}$, the intensity decreases rapidly with angle for all $P$.

The variation of polariton population with $k, N_{L P}(k)$, can be extracted from the data in Fig. 2(b), since $N_{L P} \approx I \tau_{L P}$, where $I$ is the PL intensity and $\tau_{L P}$ is the time for radiative loss from the cavity. ${ }^{13} \tau_{L P}$ is inversely proportional to the photon fraction of the polariton state, which in turn depends strongly on $k$ [see Fig. 2(c)]. The deduced variation of $N_{L P}$ with $k$, for $\Delta=-4.3 \mathrm{meV}, T=1.8$ and $34 \mathrm{~K}$, and excitation

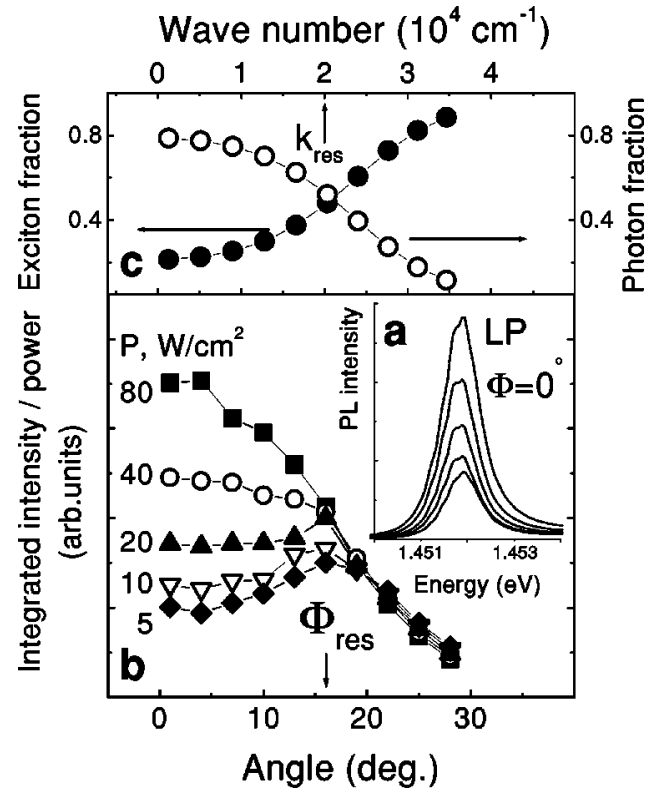

FIG. 2. (a) PL spectra normalized to the laser intensity for $P$ varying from 5 to $80 \mathrm{~W} / \mathrm{cm}^{2}$. (b) Integrated LP emission intensity divided by the laser power versus angle for detuning $\Delta=-4.3 \mathrm{meV}$. (c) Photon and exciton fractions of the polariton states for $\Delta=-4.3$ meV.

powers from 5 to $80 \mathrm{~W} / \mathrm{cm}^{2}$, is presented in Fig. 3. $N_{L P}(k)$ is very different at high and low excitation powers. At $T$ $=34 \mathrm{~K}$ [Fig. 3(a)] and low $P$, the population increases strongly with increasing $k$ for $k<k_{\text {res }}=\omega / c \sin \Phi_{\text {res }}$, and is approximately constant for $k>k_{\text {res }}\left(\approx 2 \times 10^{4} \mathrm{~cm}^{-1}\right)$. These results constitute a clear observation of the relaxation bottleneck: photocreated excitons relax to the high $k$ exciton states $\left(k>k_{\text {res }}\right)$ and then scatter with low efficiency to low $k$. With increasing $P$, a strong superlinear increase in population is found for $k<k_{\text {res }}$. At the same time, sublinear behavior is found at higher $k \geqslant k_{\text {res }}$. The overall form of the distribution changes very markedly with increasing $P$ : the growth with $k$ at low $P$ is replaced by a monotonic decrease at higher densities.

Similar behavior is observed at low $T=1.8 \mathrm{~K}$ [Fig. 3(b)]. The main difference is that at small $P$, the polariton population at $k<k_{\text {res }}$, relative to that at $k>k_{\text {res }}$, is even smaller than at high $T$. At $P=5 \mathrm{~W} / \mathrm{cm}^{2}$, the ratio of the filling of low to high $\mathrm{k}$ states is close to 3 for $T=34 \mathrm{~K}$, while it is around 6 for $T=1.8 \mathrm{~K}$. Also, the superlinear behavior, although clearly present, is less pronounced than at $34 \mathrm{~K}$.

Several important observations from the above experiments should be emphasized: (i) weak relaxation into small $k$ polariton states (the bottleneck effect) is observed at low $P$; (ii) the bottleneck is suppressed at high $P$ in the regime of the superlinear increase of PL intensity; (iii) the suppression is more pronounced at high $T$; (iv) a sublinear increase of PL intensity with $P$ is found for states with large exciton fraction at $k>k_{\text {res }}$.

We have carried out similar experiments for several different detunings $\Delta$ from +4 to $-22 \mathrm{meV}$. For zero and positive $\Delta$, the LP emission intensity peaks around $\Phi=0^{\circ}$ at all powers. For negative $\Delta$, the maxima of the PL intensity at low $P$ are always found close to $\Phi_{\text {res }}$. The bottleneck is thus 


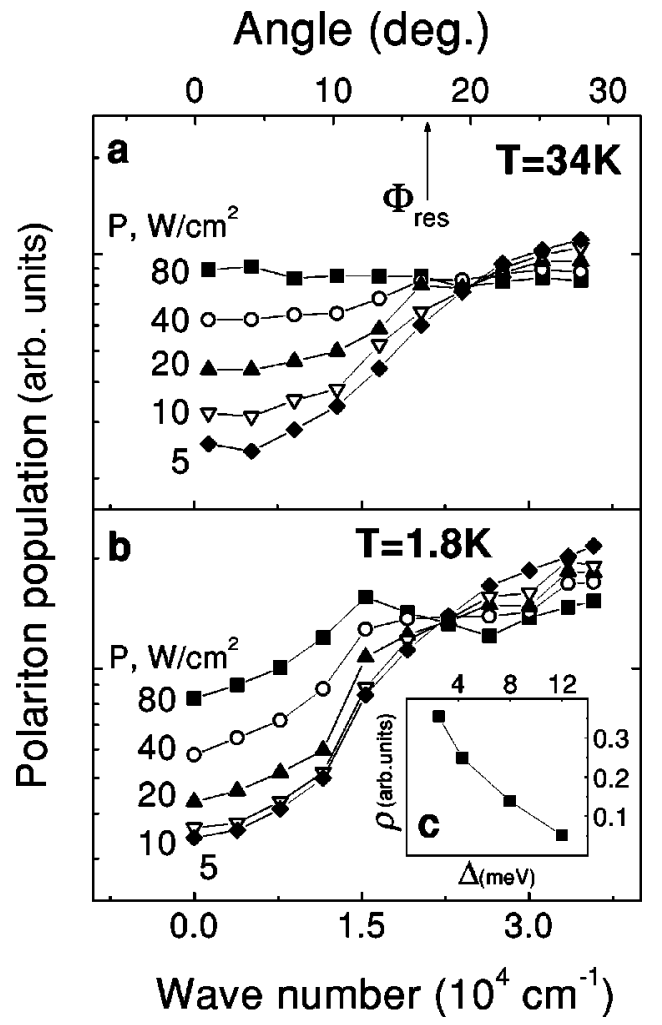

FIG. 3. Polariton population distribution for different excitation densities (a) $T=34 \mathrm{~K}$, (b) $T=1.8 \mathrm{~K}$. (c) Ratio of polariton populations $\rho=N_{L P}(0) / N_{L P}\left(k_{\text {res }}\right)$ versus $\Delta$ for low excitation and $T$ $=1.8 \mathrm{~K}$.

observed only for negative detunings, with the size of the low $k$ depletion being stronger for larger negative $\Delta$, as expected from the decreasing exciton fraction of the low $k$ states. This is shown in Fig. 3(c) where the ratio $\rho$ $=N_{L P}(0) / N_{L P}\left(k_{\text {res }}\right)$ is plotted versus $\Delta$ for $T=1.8 \mathrm{~K} ; \rho$ decreases by 6 as the detuning increases from -0.4 to $-2 \Omega$.

There have been several calculations of the polariton distribution expected under conditions of nonresonant excitation. ${ }^{4,5}$ In agreement with our observations, a bottleneck for negative $\Delta$ is predicted, but with magnitude (ratio of high $k$ to $k=0$ population) for, e.g., $\Delta=-2 \Omega$ of $\sim 10^{4}$, as opposed to less than a factor of 10 in Fig. 3. The stronger depletions predicted in the theory result in a slight dip in the calculated $k=0 \mathrm{PL}$ even at $\Delta=0$. We suggest that these disagreements arise due to the omission of disorder, freecarrier-exciton interactions and also scattering into freecarrier states (ionization) in the theory. All these processes are likely to lead to a more rapid population of the low $k$ states, and thus to a smaller amplitude of the bottleneck, which becomes negligible at zero detuning.

To investigate likely mechanisms which lead to the suppressionn of the bottleneck with increasing $P$, we analyzed the data for different detunings and temperatures. In Fig. $4(\mathrm{a})$, the ratio $I_{L P}\left(P=80 \mathrm{~W} / \mathrm{cm}^{2}\right) / I_{L P}\left(P=5 \mathrm{~W} / \mathrm{cm}^{2}\right)$ which expresses the size of the nonlinearity for a particular state, is plotted versus energy separation $\Delta E$ from the uncoupled exciton level [the definition of $\Delta E$ is shown schematically in Fig. 4(b)]. Remarkably, a clear peak in the superlinear behavior is observed at $\Delta E \approx 7 \mathrm{meV}$ for all detunings and temperatures.

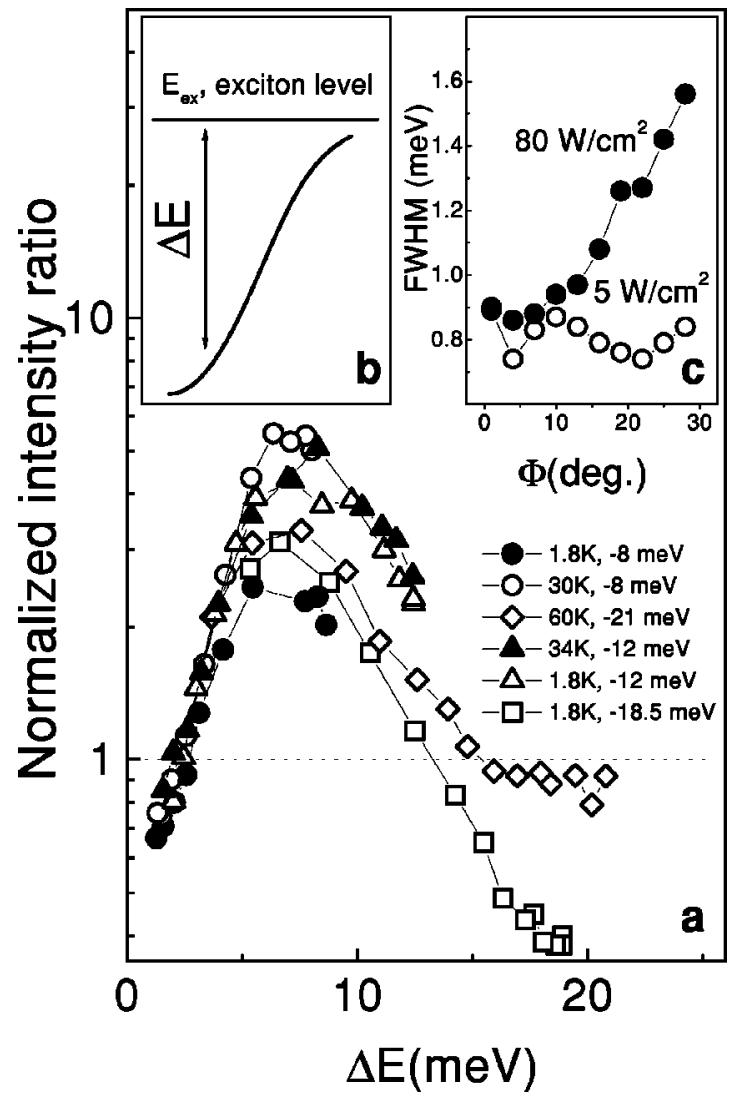

FIG. 4. (a) The ratio of normalized integrated intensities of LP emission peaks for high and low power for several negative $\Delta$ and different temperatures (indicated on the plot) versus energy separation from the exciton reservoir $(\Delta E)$. (b) Schematic definition of $\Delta E$. (c) Angular dependence of LP PL linewidth for high (solid symbols) and low (open symbols) excitation power for $\Delta=-4.3$ $\mathrm{meV}$.

The scattering maximum at $\Delta E \approx 7 \mathrm{meV}$ cannot be explained by a single acoustic phonon process since the maximum energy transferred is $\sim 1 \mathrm{meV}^{4,5}$ multiphonon processes are also very unlikely due to the fast radiative escape of lower branch polaritons. On the contrary, polaritonpolariton, polariton-exciton, and exciton-exciton scattering processes provide a variety of possibilities for singlescattering relaxation ${ }^{5}$ with larger energy transfer. The $7 \mathrm{meV}$ energy is in good agreement with that associated with two likely scattering processes: (i) exciton-exciton collisions, in which one of the excitons is scattered to free particle states and the second scatters into the strongly coupled regime (i.e., the process $X+X \rightarrow L P+e+h$, where $X, e, h$ are exciton, free electron, and hole, respectively). The exciton binding energy in a $10 \mathrm{~nm} \mathrm{In}_{0.06} \mathrm{Ga}_{0.94} \mathrm{As} / \mathrm{GaAs} \mathrm{QW}$ is $\sim 8 \mathrm{meV}$, close to the $\Delta E \approx 7 \mathrm{meV}$ value. ${ }^{14}$ (ii) A similar process to that in (i), but with the first exciton scattering from the strongly populated lower branch around $\Phi_{\text {res }}$ to the upper branch, with energy transfer given by the energy splitting between the polariton branches of $\Omega \approx 6 \mathrm{meV}$, the second exciton again scattering to the observable low $k$, LP states. Further theoretical effort is required to distinguish between these two possibilities.

There are several supporting pieces of evidence for the role of exciton-exciton scattering in populating the low $k$ 
states. First, we observe significant suppression of the bottleneck for powers greater than $\sim 20 \mathrm{~W} / \mathrm{cm}^{2}$. Assuming a lifetime of 200 psec, this corresponds to an exciton density of $\sim 1 \times 10^{9} \mathrm{~cm}^{-2}$, consistent with the density at which exciton-exciton collisions are expected to be significant $\left(\geqslant 10^{9} \mathrm{~cm}^{-2}\right){ }^{5}$ Second, exciton-exciton scattering into the LP branch has been predicted ${ }^{5}$ to increase rapidly with temperature up to $15-20 \mathrm{~K}$ (the increased spread in energy of reservoir excitons with increasing $T$ makes it easier to conserve energy and momentum), consistent with the results in Fig. 3, while phonon (emission) scattering to the LP states is calculated to be only weakly dependent on $T$.

Third, analysis of the emission from LP states with $k$ $\geqslant k_{\text {res }}$ also provides evidence for the occurrence of excitonexciton scattering. The population of these states, filled very effectively in the low excitation regime, varies sublinearly with increasing $P$ in Fig. 3. In order to demonstrate the importance of interparticle scattering for such states the angular dependence of the LP emission linewidth is shown in Fig. 4(c) for two excitation densities, at $\Delta=-4.3 \mathrm{meV}$ and $T$ $=34 \mathrm{~K}$. At low $P=5 \mathrm{~W} / \mathrm{cm}^{2}$, the linewidth is almost independent of angle $(0.8 \pm 0.06 \mathrm{meV})$. However, at high power above $\Phi \approx \Phi_{\text {res }}$, the width increases rapidly and reaches 1.6 $\mathrm{meV}$, almost twice the value at low density (the low $k$ linewidth is unchanged). We ascribe this additional broadening to strong polariton (exciton)-polariton interaction. The sublinear behavior of the PL intensity at $\Phi \geqslant \Phi_{\text {res }}[\Delta E$ $\leqslant 2.5 \mathrm{meV}$ in Fig. 4(a)] is then explained by enhanced scattering out of these states by interparticle collisions, with some of these excitons scattering to populate the lower $k$ states.

Due to the very low DOS at small $k$, filling factors close to unity are expected at the powers $\left(\sim 10^{9} \mathrm{~cm}^{-2}\right)$ employed in the present work. As a result of the bosonic character of the polaritons, stimulated scattering is then expected. ${ }^{10-12}$ In particular, in Ref. 10 the observation of superlinear emission from the LP branch, similar to that reported here, was well explained by the occurrence of stimulated scattering within a three-level rate equation model including single-phonon emission. ${ }^{15}$ It is clear that stimulated processes will enhance the quenching of the bottleneck. However, we have no direct evidence for stimulation from the results presented here: (spontaneous) exciton-exciton scattering, itself a nonlinear process, is sufficient to explain the results.

In conclusion, a strong relaxation bottleneck has been observed for the lower polariton branch in a semiconductor microcavity. Suppression of the bottleneck is found in the regime of superlinear emission which occurs with increasing power. The observations indicate that polariton-exciton interactions play a significant role in suppression of the bottleneck. Although qualitative agreement with theoretical predictions of the polariton distribution is found, disagreements remain, probably due to the omission of disorder scattering and existence of a free-carrier channel in the calculations.

We thank V. D. Kulakovskii for fruitful discussions. Financial support from EPSRC, the Royal Society, INTAS, and RFBR is gratefully acknowledged.
${ }^{1}$ C. Weisbuch et al., Phys. Rev. Lett. 69, 3314 (1992).

${ }^{2}$ J. Bloch and J. Y. Marzin, Phys. Rev. B 56, 2103 (1997); F. Tassone et al., ibid. 53, R7642 (1996).

${ }^{3}$ R. Houdre et al., Phys. Rev. Lett. 73, 2043 (1994).

${ }^{4}$ F. Tassone et al., Phys. Rev. B 56, 7554 (1997).

${ }^{5}$ F. Tassone and Y. Yamamoto, Phys. Rev. B 59, 10830 (1999).

${ }^{6}$ J. Toyazawa, Suppl. Prog. Theor. Phys. 12, 111 (1959); U. Heim and P. Weisner, Phys. Rev. Lett. 30, 1205 (1973).

${ }^{7}$ M. S. Skolnick, T. A. Fisher, and D. M. Whittaker, Semicond. Sci. Technol. 13, 645 (1998).

${ }^{8}$ M. Muller, J. Bleuse, R. Andre, and H. Ulmer-Tuffigo, Physica B 272, 476 (1999).

${ }^{9}$ In this superlinear regime, final state stimulation of the scattering has recently been reported in Refs. 10-12.

${ }^{10}$ P. Senellart and J. Bloch, Phys. Rev. Lett. 82, 1233 (1998).

${ }^{11}$ Le Si Dang et al., Phys. Rev. Lett. 81, 3920 (1998).

${ }^{12}$ A. I. Tartakovskii et al., Phys. Rev. B 60, R11 293 (1999).
${ }^{13}$ The population $N_{L P}(k)$ is related to the population in the measured angular range $\left(<2^{\circ}\right), \quad N_{L P}(\Phi)$, by $N_{L P}(k)$ $\sim N_{L P}(\Phi) / \cos (\Phi)$. The small $1 / \cos (\Phi)$ correction is applied to the data plotted in Fig. 3.

${ }^{14}$ M. K. Saker, D. M. Whittaker, M. S. Skolnick, M. T. Emeny, and C. R. Whitehouse, Phys. Rev. B 43, 4945 (1991).

${ }^{15}$ A clear observation of a stimulated polariton-polariton scattering process in MC's has recently been reported in ultrafast pumpprobe experiments. See P. N. Savvidis, J. J. Baumberg, R. M. Stevenson, M. S. Skolnick, D. M. Whittaker, and J. S. Roberts, Phys. Rev. Lett. 84, 1547 (2000). Also, experimental evidence of stimulated scattering of excitons into polariton states has been obtained; R. Huang, F. Tassone, and Y. Yamamoto, Phys. Rev. B 61, R7854 (2000). The stimulated processes identified in these works involve polariton-polariton and exciton-exciton scattering, respectively, consistent with the present conclusions. 